\title{
Preanesthetic Ultrasonography Assessment of Inferior Vena Cava Diameter in the Supine Position, Left Lateral Tilt Position, and with the Left Uterine Displacement Maneuver in Full-Term Pregnant Women: A Randomized Cross-Over Design Study
}

\author{
Tomonori Furuya \\ Nihon University School of Medicine \\ Noriya Hirose ( $\nabla$ hirose.noriya@nihon-u.ac.jp ) \\ Nihon University School of Medicine \\ Hanae Sato \\ Nihon University School of Medicine \\ Risa Niikura \\ Nihon University School of Medicine \\ Miho Kijima \\ Nihon University School of Medicine \\ Takahiro Suzuki \\ Nihon University School of Medicine
}

\section{Research Article}

Keywords: Pregnancy, Cesarean section, Inferior vena cava, Ultrasonography

Posted Date: September 7th, 2021

DOI: https://doi.org/10.21203/rs.3.rs-817625/v1

License: (c) (i) This work is licensed under a Creative Commons Attribution 4.0 International License.

Read Full License 


\section{Abstract}

\section{Background}

We used preanesthetic ultrasonography evaluation to study the effectiveness of left lateral tilt position (LLT) and the left uterine displacement maneuver (LUD) on increasing inferior vena cava diameter (IVCD) in healthy full-term pregnant women, using a randomized, single blinded, cross-over design study.

\section{Methods}

Twenty-two parturients scheduled for elective cesarean section under spinal anesthesia were recruited. All patients were sequentially placed in the supine position (SPN), LLT and with LUD before induction of spinal anesthesia. Indices of IVCD, measured by subxiphoid ultrasonography, including maximum IVCD (IVCDmax), minimum IVCD (IVCDmin) and collapsibility index (CI), and hemodynamic parameters, such as heart rate and blood pressure, were recorded in each of the postures. Mean or median values of all measurements were compared among the postures.

Results

The mean values of IVCDmax observed with both LLT and LUD were significantly larger than those in the SPN, respectively (SPN vs. LLT: $P<0.05$, SPN vs. LUD: $P<0.01$ ), although there were no significant differences between IVCDmax with LLT and LUD. There were no significant differences in IVCDmin, $\mathrm{Cl}$ and hemodynamic parameters between any of the postures. IVCDmax was highest with LUD in 11 patients (55\%), in the LLT position in seven patients (35\%) and in the SPN in two patients (10\%).

\section{Conclusions}

LLT and LUD might be equally effective in enlarging the narrowed IVCD as compared to SPN. However, both LLT and LUD might not necessarily be appropriate treatment to relieve IVC compression in some cases.

Trial registration

This study was registered in the "UMIN Clinical Trials Registry" (ID no.: 000024344, date. 10th October 2016).

\section{Introduction}

Left lateral tilt position (LLT) and the manual left uterine displacement maneuver (LUD) are commonly applied in pregnant women undergoing cesarean section, to attenuate hypotension secondary to inferior vena cava (IVC) compression [1-4]. In fact, it has been demonstrated that cardiac output and stroke volume are greater in parturients placed on an operating table tilted 15 degrees to the left as LLT, when compared with those in the supine position (SPN) $[5,6]$, LUD has also been recommended in practice 
guidelines for obstetric anesthesia, ${ }^{4}$ since it is effective in reducing both the incidence of hypotension and administration of vasopressors during spinal anesthesia $[7,8]$. However, only few studies have directly evaluated the effect of posture on alleviation of IVC compression by using IVC diameter (IVCD) as an index, one of which has suggested that the effect of LLT on enlargement of the IVCD is inconsistent, with differences between individual patients [9].

In this cross-over design study, we pre-anesthetically measured IVCD using ultrasonography in each posture, including the SPN, LLT and with LUD, in full-term pregnant women, to quantitatively evaluate the effectiveness of LLT and LUD on alleviation of IVC compression, and to evaluate the presence of interindividual differences among patients in the effect of posture on IVCD. We hypothesized that the IVCD with both LLT and LUD would be larger than that in SPN. Additionally, we hypothesized that due to interindividual differences in the IVCD with different postures, preanesthetic assessment of IVCD in different postures might help us to estimate which is the best posture to maintain blood pressure during spinal anesthesia in individual patients.

\section{Methods}

This randomized, single blinded, cross-over design study was approved by the Hospital Ethics Committee on Human Rights in Clinical Trials and Research of Nihon University Hospital (Tokyo, Japan), and was registered in the "UMIN Clinical Trials Registry" (ID no.: 000024344). Our study adheres to CONSORT guidelines.

\section{Study participants}

We obtained written informed consent from all patients. Twenty-two full-term pregnant patients, aged 20 years old or older, American Society of Anesthesiologists (ASA) physical status II, who were scheduled for non-emergency cesarean section under spinal anesthesia were included in this study. Exclusion criteria included preeclampsia, pregnancy-induced hypertension, diabetes mellitus, and obesity (body mass index $\left.>30 \mathrm{~kg} / \mathrm{m}^{2}\right)$.

All patients fasted overnight and were given an infusion of $500 \mathrm{ml}$ of crystalloid solution in the ward within an hour before their arrival at the operating room. After arriving at the operating room, patients were connected to standard monitors using ECG electrodes, an arm cuff on the right arm for noninvasive blood pressure measurement, and a pulse oximeter $\left(\mathrm{SpO}_{2}\right)$ probe on the fingertip (Life Scope ${ }^{\circledR}$, Nihon Kohden Corp., Tokyo, Japan). IVCD indices were measured by ultrasonography (C60X, 2 to $5 \mathrm{MHz}$ convex probe; MicroMaxx ${ }^{\circledR}$, SonoSite, Washington, USA) in each posture in a predetermined order with the numbered container method (Fig. 1.). In the SPN, patients lay face up on an operating table fixed perfectly horizontal; LLT was achieved by tilting the operating table 15 degrees to the left; LUD was accomplished by the method described by P. Kundra, et al [10], in which an attending anesthetist stands on the left side of the patient, and retracts the right border of the patient's uterus upward and to the left, resulting in the uterus being displaced approximately $3-5 \mathrm{~cm}$ to the left of the midline. Patients were maintained in each 
posture for 5 min, following which IVCD and hemodynamic measurements, including heart rate (HR) and blood pressure (BP) were measured and recorded. A crystalloid solution was infused at the rate of 1 $\mathrm{ml} / \mathrm{kg} / \mathrm{h}$ throughout the study. After the experiment, patients received standard combined spinal-epidural anesthesia for cesarean section.

\section{Ultrasonography}

Two attending anesthetists who measured IVCD had experienced measurement of IVCD using ultrasonography, according to American Society of Echocardiography guidelines [11], more than 50 times. They exclusively deported to visualize the IVC and measure the IVCD, respectively. The IVC was visualized in the subxiphoid window under ultrasonography using a convex probe in the long-axis view. Pulse wave Doppler was used to differentiate the IVC from the aorta. The IVCD was measured using M-mode imaging at the place that was 20 to $30 \mathrm{~mm}$ distal to the junction between right atrium and inferior vena cava. We directly measured and recorded the largest and smallest values of the IVCD by ultrasonography, i.e. IVCDmax and IVCDmin, and estimated the value of respiratory variation in IVCD to indicate the collapsibility index (CI). IVCDmax and IVCDmin were respectively defined as delineated maximum diameters of the IVC in the expiratory and inspiratory phase, respectively. While the IVCD was measured by ultrasonography in each posture, patients held their steady breathing. $\mathrm{Cl}$ was calculated using the following formula: $\mathrm{Cl}(\%)=$ [(IVCDmax - IVCDmin) / IVCDmax $\times$ × 100. These corrected data were blinded by using the prospective randomized open blinded end-point (PROBE) method, and were analyzed by the triers other than measurers. The values of these data were compared between the three postures in each patient. Additionally, the distribution of the measured values of IVCDmax in each posture was described in each patient, since IVCDmax might be a reliable indicator of intravascular volume status [12-14].

\section{Statistically analysis}

Sample size in this study was based on the fact that one of the primary outcomes in this study was the difference in the mean value of IVCDmax between the three postures. Power analysis was based on our preliminary test, where the mean values (SD) of IVCDmax in SPN, LLT and LUD were 15.4 (3.7), 16.2 (3.0) and 19.2 (2.8) $\mathrm{mm}$, respectively. Thus, the sample size was determined as 18 patients for comparison of mean values of IVCDmax among the postures, using a priori repeated measure analysis of variance (ANOVA) with effect size $(f)=0.44, a=0.05$ and a power of 0.80 . In consideration of potential dropouts, we enrolled 22 patients in this study. The patients characteristics and clinical data were presented as means (SD), medians (quartile deviation: QD) or number of cases (\%). IVCD and hemodynamic parameters, i.e. IVCDmax, IVCDmin, $\mathrm{Cl}, \mathrm{BP}$ (systolic BP; sBP, diastolic BP; dBP) and HR, in each posture were expressed as means (SD) or medians (QD), and were compared between postures by the TukeyKramer or Steel-Dwass multiple comparison test. Statistical analysis was performed using JMP version 9 (SAS Institute, Cary, NC, USA). P-values $<0.05$ were considered significant.

\section{Results}


The outcomes of 20 patients were analyzed and reported, since the data of two patients whose IVC image on ultrasonography was obscure were excluded from the study (Fig. 1). The patients' characteristics and clinical data are shown in Table 1. None of the cases experienced critical changes in hemodynamic parameters or subjective symptoms.

IVC parameters and hemodynamics in the three postures are presented in Tables 2 and 3. Mean values of IVCDmax observed with both LLT and LUD were significantly higher than that in SPN (SPN vs. LLT: $P=$ $0.0353 ; 95 \% \mathrm{Cl}(0.17-5.91)$, SPN vs. LUD: $P=0.008 ; 95 \% \mathrm{Cl}(0.84-6.58))$. The mean value of IVCDmax observed with LUD was larger than that in LLT, although the difference was not statistically significant. There was also no statistically significant difference between the postures in terms of IVCDmin, $\mathrm{Cl}, \mathrm{BP}$ and HR. The values of IVCDmax in each posture in all patients are shown in Fig. 2. IVCDmax was highest in the LUD in 11 patients (55\%), in the LLT in 7 patients (35\%) and in the SPN in 2 patients $(10 \%)$.

\section{Discussion}

The present study showed that the mean values of IVCDmax observed with both LLT and LUD were significantly larger than that in SPN. Based on reports that IVCDmax measured by ultrasonography is a reliable index of IVCD [12-14], the present results suggest that both LLT and LUD can effectively relieve compression of the IVCD by the gravid uterus that occurs in SPN in full-term pregnant patients.

Additionally, the fact that a few patients (10\%) demonstrated the largest IVCDmax in SPN in the present study cautions us that both LLT and LUD are not necessarily appropriate treatment for relieving IVCD compression in pregnant women.

Based on previous reports that showed that application of LLT or the LUD maneuver ameliorated the decrease in maternal blood pressure and enhanced vasopressor requirements observed in the supine position $[5,6,10]$, it is believed that both LLT and LUD lead to an increase in venous return to the right atrium via relief of the SPN-induced IVC compression. A previous report showed that IVCDmax measured by ultrasonography might be a reliable indicator of intravascular volume status, since it is almost directly proportional to central venous pressure $[11,15]$. A previous study that quantitatively evaluated IVCD using ultrasonography, as in our study, demonstrated that the IVCDmax observed with both LLT and right lateral tilt positioning were significantly larger than that observed in SPN [9]. Conversely, several studies showed that the angle of LLT by $15^{\circ}$ and $20^{\circ}$ had no effect on IVCD enlargement $[16,17]$. Another study also showed that the IVCD measured by magnetic resonance imaging significantly increased with LLT by $30^{\circ}$ and $45^{\circ}$ compared with that in SPN, although a $15^{\circ}$ LLT had no significant effect [18]. Additionally, it has been demonstrated that LLT might lead to narrowing of the IVCD due to compression of the IVC by the liver [19]. However, while there is no report evaluating the effect of LUD on enlargement of the IVCD using quantitative measurement methods, some studies demonstrated that LUD was more effective in maintaining hemodynamics during spinal anesthesia than LLT [10]. Considering these above facts, the present results showing that IVCDmax was significantly larger with LLT and LUD than that in SPN suggested that both LLT and LUD are effective in increasing blood flow volume in the IVC due to enlargement of the IVCD. Also, in the present study, more patients had the largest IVCDmax with LUD as 
compared to LLT although the mean values of IVCDmax with LLT and LUD were statistically equivalent, suggesting that LUD might be a more reliable method to relieve IVCD compression than LLT.

There were no statistically significant differences in measured IVCD parameters other than IVCDmax in the three postures in this study. In ultrasonography for the IVC, the combined evaluation of IVCDmax and $\mathrm{Cl}$ value, calculated as the difference between IVCDmax and IVCDmin, which denotes respiratory variation in IVCD, can estimate the status of circulating blood volume and right atrial pressure (RAP), as below [11]. It is estimated that RAP might decrease to $<5 \mathrm{mmHg}$ and hypovolemia may develop when IVCDmax $\leq 21$ $\mathrm{mm}$ and $\mathrm{Cl}>50 \%$, and RAP may increase to $>15 \mathrm{mmHg}$ and hypervolemia may develop when IVCDmax > $21 \mathrm{~mm}$ and $\mathrm{Cl}<50 \%$. Therefore, since the IVCDmax and $\mathrm{Cl}$ in each posture of all patients observed in the present study did not fulfill the above condition, circulating blood volume in all the patients might have been within the normal range and their RAP might have been suitably maintained in each posture. Also considering the fact that there were no significant differences in values of $\mathrm{BP}$ and $\mathrm{HR}$ among the three postures, the effect of both LLT and LUD on increase in IVC blood flow secondary to enlargement of IVCDmax might be slight, which would explain the lack of significant improvement in the hemodynamic decline seen in the SPN by adoption of LLT or LUD. However, the measured values in this study were not obtained under spinal anesthesia and were evaluated in healthy parturients. After induction of spinal anesthesia, blood pressure often suddenly decreases due to decrease in central blood volume [20, 21], and both LLT and LUD suppress this decrease in blood pressure in clinical settings [5, 6, 10]. Additionally, there exists a phenomenon whereby parturients with pre-existing and pregnancy-induced hypertension have a greater tendency to develop hypovolemia [22]. Considering the above facts, it is easy to assume that the effectiveness of LLT and LUD on enlargement of IVCD observed in this study would be more remarkable in anesthetized and/or parturients with complications. Therefore, further studies are needed in order to define the real effect of LLT and the LUD maneuver on IVCD measured using ultrasonography in actual clinical settings.

The present study also showed that more patients had the largest IVCDmax with LUD as compared to LLT and SPN, as seen in the individual evaluations of patients. This order of incidences of the largest observed values of IVCDmax were not inconsistent with the results of the mean values of IVCDmax in the respective postures. Meanwhile, it should be noted that the effectiveness of the three postures on IVCDmax enlargement was not uniform between patients, as indicated by the fact that there were two patients $(10 \%)$ in whom the largest IVCDmax was found in SPN. This suggests that both LLT and LUD are not necessarily appropriate treatment to relieve compression of the IVC in some cases. Although the reason for the individual differences in the effects of each posture on IVCD is still unclear, this could be due to variations in certain factors, such as placental site, size of the uterus and uterine adnexa, and collateral circulation via the vertebral venous system $[9,23]$. Ultrasonography for evaluation of the IVC can be performed non-invasively and comparatively easily, and economically. ${ }^{12}$ In fact, we were able to measure IVCDmax and IVCDmin in all postures within a total of about 20 min in almost all our patients, although we were unable to visualize the IVC clearly in two patients. Therefore, ultrasonography for assessment of the IVC before induction of spinal anesthesia can be easily performed not only in the 
operating room, but also at the patient's bedside in the ward as a point-of-care method [24, 25]. Preanesthetic ultrasonography assessment of the IVC might help us to preoperatively estimate which is the best posture to maintain IVCD during spinal anesthesia for cesarean section in individual patients.

There are several limitations to this study. First, the present data were obtained from patients who were not under spinal anesthesia. Since it is possible that the effect of each posture on IVCD, as described in the study, might be different in patients under spinal anesthesia, the part of our discussion related to the effects of these postures on IVCD under spinal anesthesia is only speculative. Second, it is desirable that ultrasonography for IVC assessment should be performed under standardized respiratory conditions in order to obtain accurate values of IVCD $[26,27]$. The measured values of IVCD parameters in this study might have been affected by variations in respiratory conditions in the three postures in each patient, even though we urged all patients to hold their steady breathing during ultrasonography in all three postures.

\section{Conclusions}

We used ultrasonography to evaluate the effects of three postures, SPN, LLT and LUD, on IVCD in healthy full-term pregnant patients, using a cross-over design. LLT and LUD might be equally effective in preventing compression of the IVCD in SPN. However, both LLT and LUD might not necessarily be appropriate treatment to relieve IVC compression during spinal anesthesia in some cases. Preanesthetic ultrasonography assessment of IVCD might help us to estimate which is the best posture to maintain blood pressure during spinal anesthesia for cesarean section in individual patients.

\section{Abbreviations}

LLT: left lateral tilt position

LUD: manual left uterine displacement maneuver

SPN: supine position

IVC: inferior vena cava

IVCD: inferior vena cava diameter

Cl: collapsibility index

IVCDmax: maximum inferior vena cava diameter

IVCDmin: minimum inferior vena cava diameter

RAP: right atrial pressure

$\mathrm{SpO}_{2}:$ pulse oximeter 
HR: heart rate

BP: blood pressure

sBP: systolic blood pressure

dBP: diastolic blood pressure

\section{Declarations}

Ethics approval and consent participate:

This study was approved by the Hospital Ethics Committee on Human Rights in Clinical Trials and Research of Nihon University Hospital (Tokyo, Japan) (ID no. RK-160913-10, date 30th September 2016)

Consent for publication: Not applicable

Availability of data and materials:

The datasets used and/or analysed during the current study are available from the corresponding author on reasonable request.

Competing interest:

The authors declare that they have no competing interests.

Funding:

This study was supported by Grant-in-Aid for Young Scientists (B) (15K20061) of Japan society for the Promotion of Science. (Furuya T.)

Authors' contributions:

TF planed the research, visualized the IVC using ultrasonography and wrote the first draft of the manuscript. NH planed and designed the study, analyzed the data and revised the first draft of the manuscript. HS recruited participants, acquired participants consent and performed the randomization and the blind procedure. RN recruited participants, acquired participants consent and analyzed the data. MK recruited participants, acquired participants consent and measured the IVCD using ultrasonography. TS designed the study, analyzed the data and revised the first draft of the manuscript. All authors read and approved the final manuscript.

Acknowledgements: Not applicable

\section{References}


1. Ker MG, Scott DB, Samuel E. Studies of the inferior vena cava in late pregnancy. Br Med J. 1964; 1: 532-3.

2. Scott DB. Inferior vena caval occlusion in late pregnancy and its importance in anaesthesia. $\mathrm{Br} J$ Anaesth. 1968; 40: 120-8.

3. Hirabayashi Y, Shimizu R, Fukuda H, Saitoh K, Igarashi T. Effects of the pregnant uterus on the extradural venous plexus in the supine and lateral positions, as determined by magnetic resonance imaging. Br J Anaesth. 1997; 78: 317-9.

4. Practice guidelines for obstetric anesthesia: An updated report by the American Society of Anesthesiologists Task Force on Obstetric Anesthesia and the Society for Obstetric Anesthesia and Perinatology. Anesthesiology. 2016; 124: 270-300.

5. Secher NJ, Arnsbo P, Andersen LH, Thomsen A. Measurements of cardiac stroke volume in various body positions in pregnancy and during Caesarean section: A comparison between thermodilution and impedance cardiography. Scand J Clin Lab Invest. 1979; 39: 569-76.

6. Lee SW, Khaw KS, Ngan Kee WD, Leung TY, Critchley LA. Haemodynamic effects from aortocaval compression at different angles of lateral tilt in non-labouring term pregnant women. $\mathrm{Br} \mathrm{J}$ Anaesth. 2012; 109: 950-6.

7. Dyer RA, Reed AR, van Dyk D, Arcache MJ, Hodges O, Lombard CJ, et al. Hemodynamic effects of ephedrine, phenylephrine, and the coadministration of phenylephrine with oxytocin during spinal anesthesia for elective cesarean delivery. Anesthesiology. 2009; 111: 753-65.

8. Chungsamarnyart $Y$, Wacharasint $P$, Carvalho $B$. Hemodynamic profiles with and without left uterine displacement: A randomized study in term pregnancies receiving subarachnoid blockade for cesarean delivery. J Clin Anesth. 2020; 64: 109796.

9. Fields JM, Catallo K, Au AK, Rotte M, Leventhal D, Weiner S, et al. Resuscitation of the pregnant patient. What is the effect of patient positioning on inferior vena cava diameter? Resuscitation. 2013; 84: 304-8.

10. Kundra P. Khanna S, Habeebullah S, Ravishankar M. Manual displacement of the uterus during Cesarean section. Anaesthesia. 2007; 62: 460-5.

11. Rudski LG, Lai WW, Afilalo J, Hua L, Handschumacher MD, Chandrasekaran K, et al. Guidelines for the echocardiographic assessment of the right heart in adults: a report from the American Society of Echocardiography endorsed by the European Association of Echocardiography, a registered branch of the European Society of Cardiology, and the Canadian Society of Echocardiography. J Am Soc Echocardiogr. 2010; 23: 685-713.

12. Brennan JM, Ronan A, Goonewardena S, Blair JE, Hammes M, Shah D, et al. Handcarried ultrasound measurement of the inferior vena cava for assessment of intravascular volume status in the outpatient hemodialysis clinic. Clin J Am Soc Nephrol. 2006; 1: 749-753.

13. Zengin S, Al B, Genc S, Yildirim C, Ercan S, Dogan M, et al. Role of inferior vena cava and right ventricular diameter in assessment of volume status: a comparative study: ultrasound and hypovolemia. Am J Emerg Med. 2013; 31: 763-7. 
14. Sonoo T, Nakamura T, Ando T, Sen K, Maeda A, Kobayashi E, et al. Prospective analysis of cardiac collapsibility of inferior vena cava using ultrasonography. J Crit Care. 2015; 30: 945-8.

15. Babaie S, Behzad A, Mohammadpour M, Reisi M. A comparison between the bedside sonographic measurements of the inferior vena cava indices and the central venous pressure while assessing the decreased intravascular volume in children. Adv Biomed Res. 2018; 7: 213-7.

16. Rees SG, Thurlow JA, Gardner IC, Scrutton MJ, Kinsella SM. Maternal cardiovascular consequences of positioning after spinal anaesthesia for Caesarean section: Left 15 degree table tilt vs. left lateral. Anaesthesia. 2002; 57: 15-20.

17. Matorras R, Tacuri C, Nieto A, Gutierrez de Terán G, Cortés J. Lack of benefits of left tilt in emergent cesarean sections: a randomized study of cardiotocography, cord acid-base status and other parameters of the mother and the fetus. J Perinat Med. 1998; 26: 284-92.

18. Higuchi H, Takagi S, Zhang K, Furui I, Ozaki M. Effect of lateral tilt angle on the volume of the abdominal aorta and inferior vena cava in pregnant and nonpregnant women determined by magnetic resonance imaging. Anesthesiology. 2015; 122: 286-93.

19. Mookadam F, Warsame TA, Yang HS, Emani UR, Appleton CP, Raslan SF. Effect of positional changes on inferior vena cava size. Eur J Echocardiogr. 2011; 12: 322-5.

20. Lee JE, George RB, Habib AS. Spinal-induced hypotension: incidence, mechanisms, prophylaxis, and management: summarizing 20 years of research. Best Pract Res Clin Anaesthesiol. 2017; 31: 57-68.

21. Ithnin F, Lim Y, Sia AT, Ocampo CE. Combined spinal epidural causes higher level of block than equivalent single-shot spinal anesthesia in elective cesarean patients. Anesthesia and Analgesia. 2006; 102: 577-80.

22. Cotton DB, Lee W, Huhta JC, Dorman KF. Hemodynamic profile of severe pregnancy-induced hypertension. Am J Obstet Gynecol. 1998; 158: 523-6.

23. Humphries A, Mirjalili SA, Tarr GP, Thompson J, Stone P. The effect of supine positioning on maternal hemodynamics during late pregnancy. J Matern Fetal Neonatal Med. 2019; 32: 3923-30.

24. Levitov A, Frankel HL, Blaivas M, Kirkpatrick AW, Su E, Evans D, et al. Guidelines for the appropriate use of bedside general and cardiac ultrasonography in the evaluation of critically ill patients-part II: cardiac ultrasonography. Crit Care Med. 2016; 44: 1206-27.

25. Ciozda W, Kedan I, Kehl DW, Zimmer R, Khandwalla R, Kimchi A. The efficacy of sonographic measurement of inferior vena cava diameter as an estimate of central venous pressure. Cardiovasc Ultrasound. 2016; 14: 33.

26. Bortolotti P, Colling D, Preau S. Inferior vena cava respiratory variations: a useful tool at bedside to guide fluid therapy in spontaneously breathing patients. Shock. 2018; 49: 235-6.

27. Airapetian N, Maizel J, Alyamani O, Mahjoub Y, Lorne E, Levrard M, et al. Does inferior vena cava respiratory variability predict fluid responsiveness in spontaneously breathing patients? Crit Care. 2015; 19: 400.

\section{Tables}


Due to technical limitations, tables are only available as a download in the Supplemental Files section.

Figures

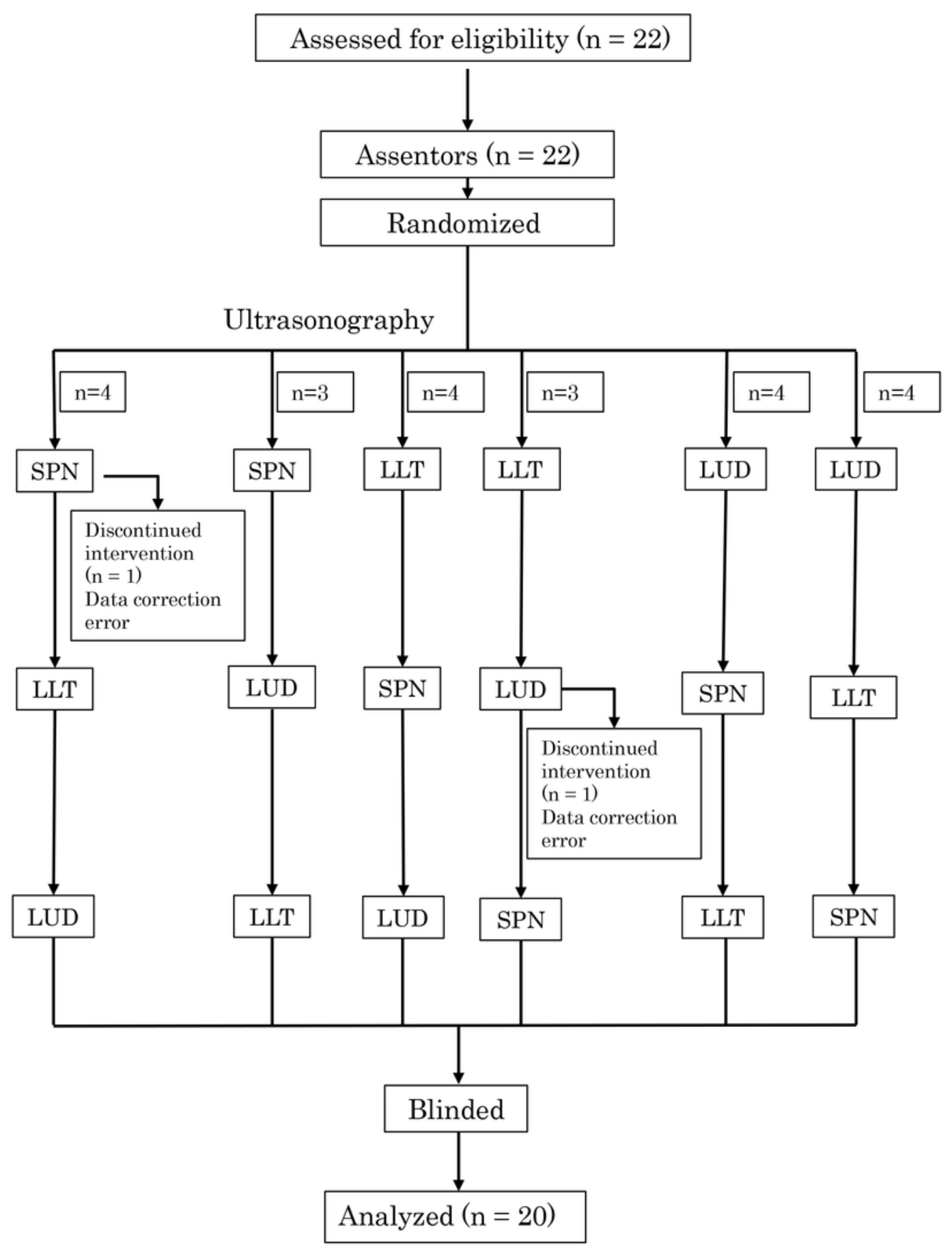

Figure 1 
Consort flow diagram. SPN; supine position, LLT; left lateral tilt position, LUD; manual left uterine displacement maneuver.

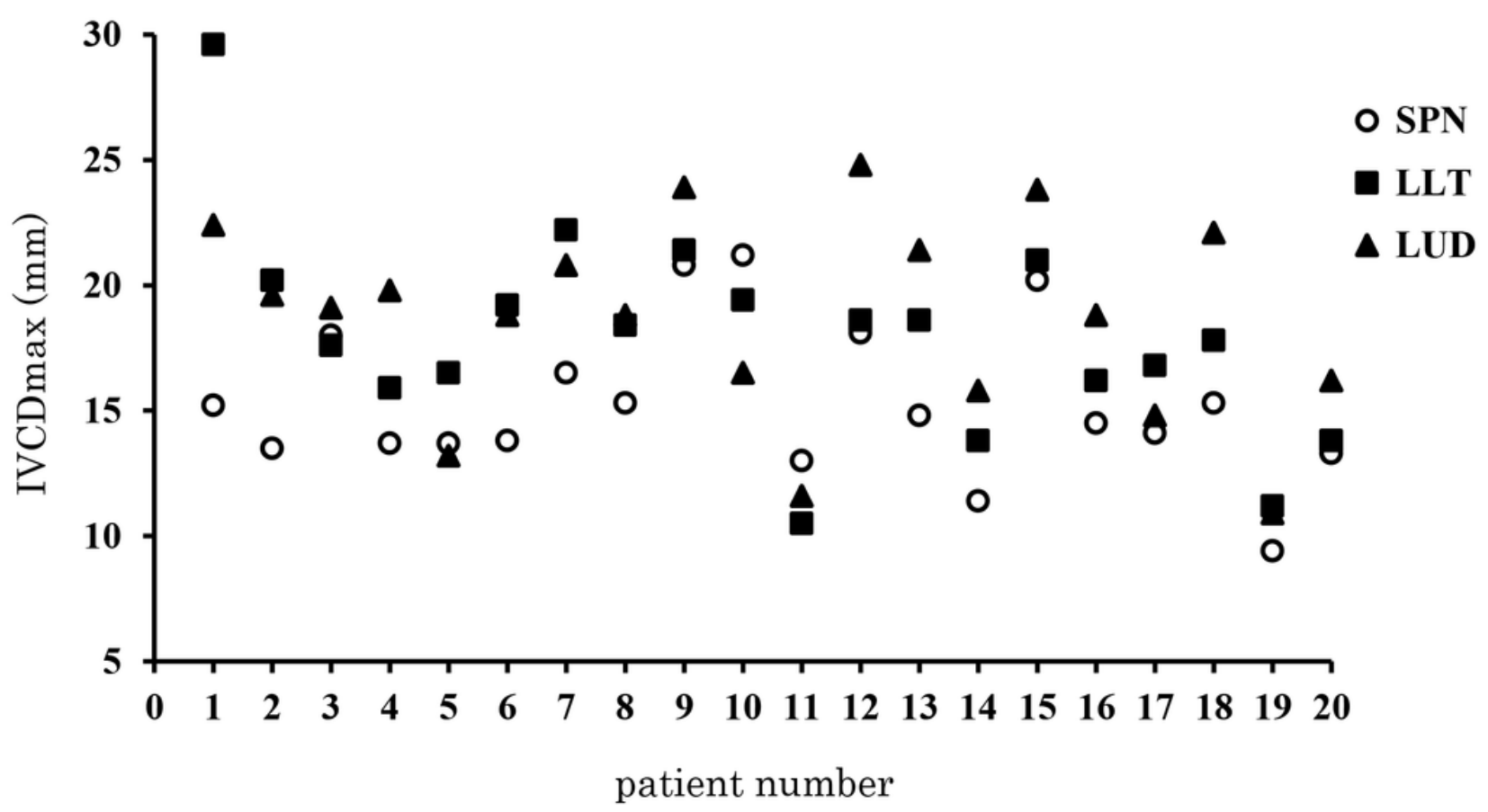

Figure 2

Values of IVCDmax in the three postures in each patient. IVCDmax; maximum inferior vena cava diameter, SPN; supine position, LLT; left lateral tilt position, LUD; manual left uterine displacement maneuver.

\section{Supplementary Files}

This is a list of supplementary files associated with this preprint. Click to download.

- FuruyaTable1.xlsx

- FuruyaTable2.xIsx

- FuruyaTable3.xlsx 